\title{
Observations on the responses of muscle to mechanical and electrical stimuli
}

\author{
J. C. MEADOWS
}

From the Department of Neurological Studies, The Middlesex Hospital, London

SUMMARY Responses to mechanical and electrical stimuli have been studied in vastus medialis in four young adults. Percussion causes an immediate, brief contraction in those muscle fibres passing beneath the site of the blow. This is accompanied by EMG activity which is propagated along the muscle fibres at a normal velocity of around $4 \mathrm{~m} / \mathrm{sec}$. The EMG activity lasts much longer than that produced by a single electrical stimulus to muscle fibres because repetitive firing occurs in some of the muscle fibres activated mechanically. This response to percussion is unaffected by nerve blockade with $2 \%$ xylocaine. Percussion close to the motor point may cause delayed fasciculation due to activation of intramuscular motor nerve fibres. This, too, is unaffected by nerve blockade. Some observations on EMG insertional activity provoked by needle movement are reported. It is concluded that muscle has a basic tendency to discharge repetitively when stimulated by mechanical means and that EMG insertional activity and the EMG response to percussion reported in this paper are both manifestations of this same tendency, which is increased in the myotonias.

It has long been known that percussion of muscle can cause a direct contraction independent of nerves ('idiomuscular contraction'). This phenomenon was described in animals by Schiff in 1858 and it was not long before it was studied in man. The major feature that was described was an immediate contraction at the percussion site, but in subjects debilitated by tuberculosis or carcinomatosis small waves of contraction were also described which radiated slowly in either direction along the muscle from the percussion site. Indeed, this response was at one time considered an important diagnostic sign in tuberculosis, although a direct relationship was subsequently disproved (Curschmann, 1905).

The early literature is discussed by Denny-Brown and Pennybacker (1938) who were the first to study the effects of percussion electrophysiologically. These authors state that in the normal person there is a localized contraction at the percussion site which is associated with tightening of the remainder of the fasciculus, both subsiding in from 1 to 5 seconds. This is accompanied by irregular, fine action currents, indicating that local, diffuse excitation has occurred'. In their normal subjects they did not report spreading waves of contraction but did see this phenomenon in a patient with wasting as part of a general cachexia from carcinoma. In this patient, the local contraction (myoedema) lasted some 10 seconds and was accompanied by the usual rapid action currents and fascicular tightening. In addition, a small transmitted wave was seen to leave the percussion site and travel along the muscle fibres. This wave travelled at about $2 \mathrm{~cm}$ per second and was unaccompanied by recordable electrical activity. Denny-Brown and Pennybacker concluded that the fully developed idiomuscular response consists of two components, an immediate twitch contraction involving the action potential mechanism and a longer lasting contraction of the muscle fibre contents which in cachectic states may be the starting point for slow spreading waves of contraction that are not accompanied by action potentials.

The nature of the 'irregular, fine action currents' described by Denny-Brown and Pennybacker cannot be deduced from their paper, written over 30 years ago and the few references to the subject since are conflicting. Floyd, Kent, and Page (1955), in a study of dystrophia myotonica, mention that in the normal subject percussion may produce 'a few motor unit potential discharges'. Again studying myotonia, Brown and Harvey (1939) described how, in the normal goat, a blow to the muscle is 'followed by a 
brief outburst of very asynchronous potentials which lasts at the most one second ... Decentralization of a muscle or degeneration of its nerve does not diminish the brief discharge which is evoked by tapping'. In contrast to these reports, Salick and Pearson (1967) were unable to record any electrical activity during the prolonged contraction resulting from a muscle tap in a patient with myxoedema. Obviously the problem may be different here, since the mechanics of the contractile process are known to be profoundly altered in hypothyroidism but these authors do not appear to have recorded EMG activity even in the early part of the response and conclude that the blow produced an 'immediate, electrically silent contraction'. However, since they recorded immediately adjacent to the percussion site, such activity might easily be lost in the movement artefact provoked by the tap.

There seemed to be a possible discrepancy between these various reports and the present study was therefore undertaken to delineate more clearly the visual and electromyographic events that occur when healthy muscle is percussed, with a view to examining the problem later in pathological states. EMG activity proved difficult to discern close to the site of percussion because of movement artefact, but could be regularly recorded at a distant point along the muscle fibres. The latency of the EMG response was found to approximate to the latency after direct electrical stimulation of muscle fibres through a needle inserted at the percussion site and corresponded to a muscle membrane conduction velocity of about $4 \mathrm{~m} / \mathrm{sec}$. However, the duration of the response was much longer than that evoked by electrical stimulation and this was finally shown to be due to repetitive discharge of some of the muscle fibres activated by percussion. It appears that the repetitive discharge so characteristic of myotonia may occur in an abbreviated form in the normal subject.

\section{METHODS}

Experiments were carried out in four healthy subjects, aged 23 to 30 . EMG recordings were made of responses to different stimuli in vastus medialis. The subject was sitting in a warm room with the knee bent to a right angle and recordings were taken from the main bulk of the muscle.

Two different recording electrodes were employed. Concentric unipolar needle electrodes (Rank Med. 150.7 and $150 \cdot 6$ ) were used in the majority of experiments and the EMG signal was displayed on the oscilloscope screen of a two channel Medelec SDC 3 EMG machine, having a frequency band of $1 \cdot 6-5,000 \mathrm{c} / \mathrm{sec}, 3 \mathrm{db}$ down. In other experiments a modified Ekstedt needle (Ekstedt, 1964; Stålberg, 1967) was used and recordings were then taken from one of its small ( $30 \mu$ diameter) recording surfaces, using a distant plate electrode as a reference. Screened $Z$ leads led from the electrode to a cathode follower and an $\stackrel{\mathbb{D}}{=}$ amplifier system similar to that described by Ekstedt. The advantage of this type of electrode in the present experi- $\stackrel{\text { ? }}{=}$ ments is that its small recording surface and high input $\widehat{0}$ impedance allow individual components of the EMG to $D$ be discerned during percussion even when many muscle fibres have been activated.

RESPONSES TO PERCUSSION These were studied following. blows with a tendon hammer to the extreme distal end of $\Rightarrow$ the muscle which, with the knee bent, is spread over the $\stackrel{?}{?}$ bony femoral condyle (Fig. 1A). The oscilloscope sweep? was triggered by the impact of the hammer causing $\underline{\sigma}$ contact between two closely separated metal strips $\overline{\bar{F}}$ attached to the shaft of the hammer, close to its head. It $\vec{\varnothing}$ was established that there was a delay of between 1.8 and $\varrho$ $3.5 \mathrm{msec}$ when triggering by this method, the duration i $^{2}$
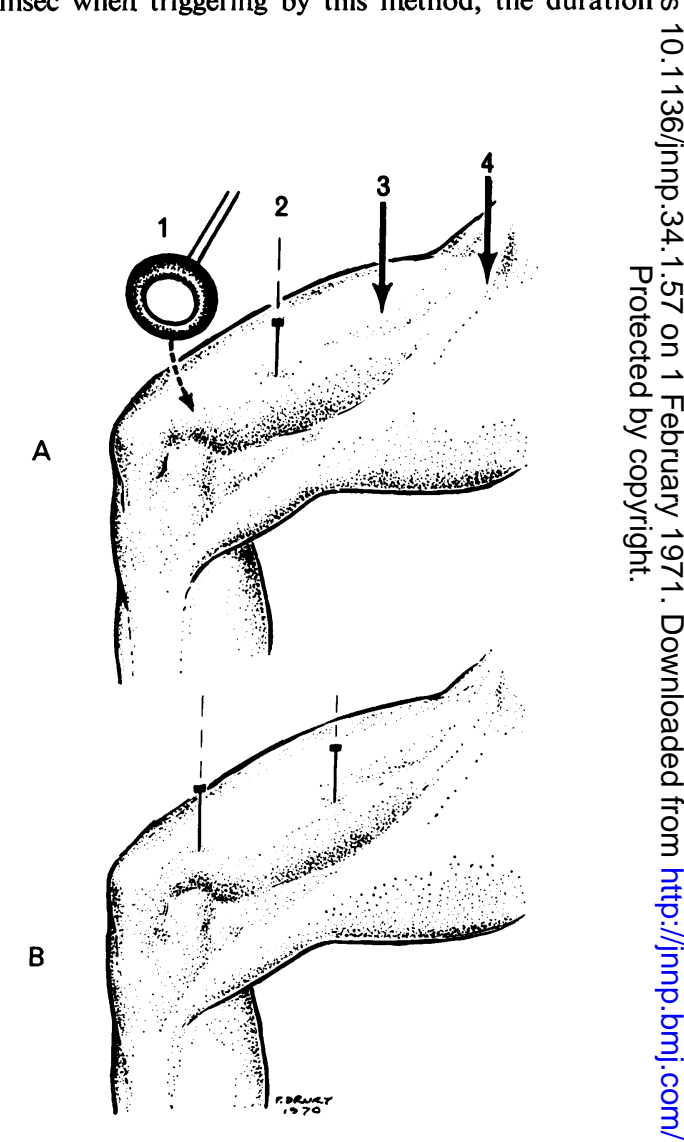

FIG. 1. A. Diagram to show percussion (I) and recording 응 (2) sites in vastus medialis. In a few experiments the nerve? to vastus medialis was blocked with xylocaine in mid-thigh (3) and the effect of the block was confirmed by stimulating the nerve above the site of the block (4). B. Diagram to N illustrate the needle positions used for measuring muscle membrane conduction velocity and for studying propagation of EMG insertional activity. 
being inversely proportional to the velocity of the blow. This short delay does not influence any of the conclusions drawn.

RESPONSES TO NEEDLE MOVEMENT These were studied by recording EMG activity both at the needle at which the activity arose and also at a second concentric needle electrode aligned along the fibres at a distance along the muscle (Fig. 1B).

MUSCLE MEMBRANE CONDUCTION VELOCITY This was measured by stimulating muscle fibres electrically through a concentric needle electrode and recording the evoked response with an identical needle placed proximally as before (Fig. 1B). As in the experiments on percussion and insertional activity, the stimulus was always as far distally as possible in the muscle, well distal to the region of the motor point where most end plates are found (Coërs and Woolf, 1959). In this way stimulation of intramuscular nerve fibres should have been avoided. The motor point of vastus medialis lies approximately three-fourths of the way down the medial side of the thigh (Walthard and Tchicaloff, 1961) and this was confirmed in each subject.

The usual procedure for measuring conduction velocity was to insert the stimulating needle and deliver small cathodal shocks $(2-25 \mathrm{~V}, 0.05 \mathrm{msec}$ every second from a Medelec S13 stimulator) to the outer casing of the needle, the anode being provided by a plate electrode strapped to the leg. A narrow strip of contraction could then be seen extending proximally from the stimulating needle. Cutaneous stimulation also occurred and the procedure was slightly painful with the larger shocks. It was comparatively easy to insert the recording electrode among the contracting fibres 6 to $12 \mathrm{~cm}$ proximal to the stimulating site, to record a brief response of sharp and usually polyphasic outline. The terminals of the stimulating needle were then changed so that the central core became the cathode and the outer casing the anode. In this way the stimulus was confined to the region of the electrode tip. Shocks of the same magnitude then produced no conscious sensation and no visible contraction could be seen except with the larger stimuli, when both needles might be seen twitching slightly with each stimulus. If an EMG response showing sharp transients (indicating close proximity of the recording electrode to the contracting fibres) was not immediately evident, slight withdrawal of the stimulating needle or minor alterations in the position of the recording electrode usually produced such a response. With shocks in the region of threshold, only one or two small diphasic potentials might then be recorded. Electrode position was of course critical, and the subject had to remain quite still during the procedure.

The distance between the tips of the two needles was measured as accurately as possible and conduction velocity for the first spike appearing at threshold stimulation was calculated.

VALIDITY OF NEEDLE STIMULATION TECHNIQUE Stålberg (1967) has questioned the validity of needle stimulation techniques that are said directly to activate muscle fibres, suggesting that the responses recorded are the result of intramuscular nerve fibre stimulation. The EMG response evoked by the present technique cannot be due to intramuscular nerve fibre stimulation for the following reason. Once the two needles had been positioned satisfactorily their roles could be reversed (taking great care not to disturb their positions) so that conduction could be studied in both directions (Fig. 2). This would not be possible if the response were due to intramuscular nerve fibre stimulation.

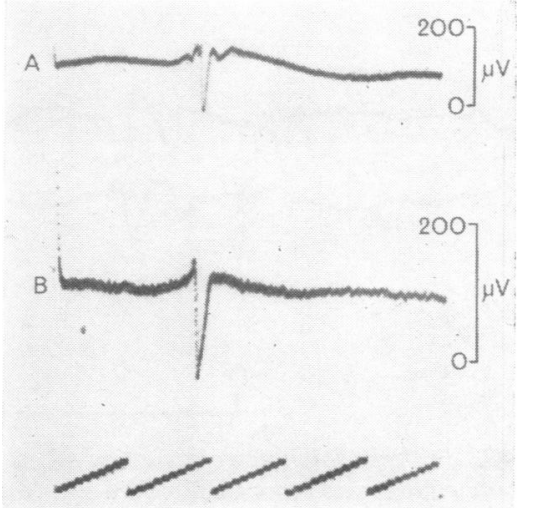

FIG. 2. A. Evoked EMG responses after stimulation through a concentric needle electrode as in Fig. 1B. Threshold activation for the first spike potential required about $3 \mathrm{~V}$. Recording distance $7.5 \mathrm{~cm}$. B. Needle terminals reversed so that stimulating electrode became recording electrode and vice versa. Time scale $10 \mathrm{msec}$ per slope. The spike in each record appeared abruptly at a critical stimulation threshold and did not alter with further increases in stimulus strength until additional potentials became superimposed upon it. The minor irregularities either side of the spike potential in record $\mathbf{A}$ represent activation of other fibres less close to the tip of the recording electrode.

There are other reasons why the stimulation technique used cannot depend upon stimulating intramuscular nerve fibres. During the initial localization procedure when large shocks were delivered through the casing of the stimulating needle, a strip of muscle could be seen contracting in a straight line between the two needles. This would not necessarily be the case if the effect was due to intramuscular nerve fibre stimulation. Once the needles were in position, careful adjustments of the stimulus intensity caused individual components to appear in an all-or-none manner and their diphasic configuration resembled fibrillation rather than motor unit potentials which would be the case with nerve fibre stimulation. If the terminals of the two needles were then connected to separate recording channels (taking great care not to disturb the position of the needles), near-synchronous potentials could be recorded on slight voluntary contraction (Fig. 3), as would be expected if the needles 

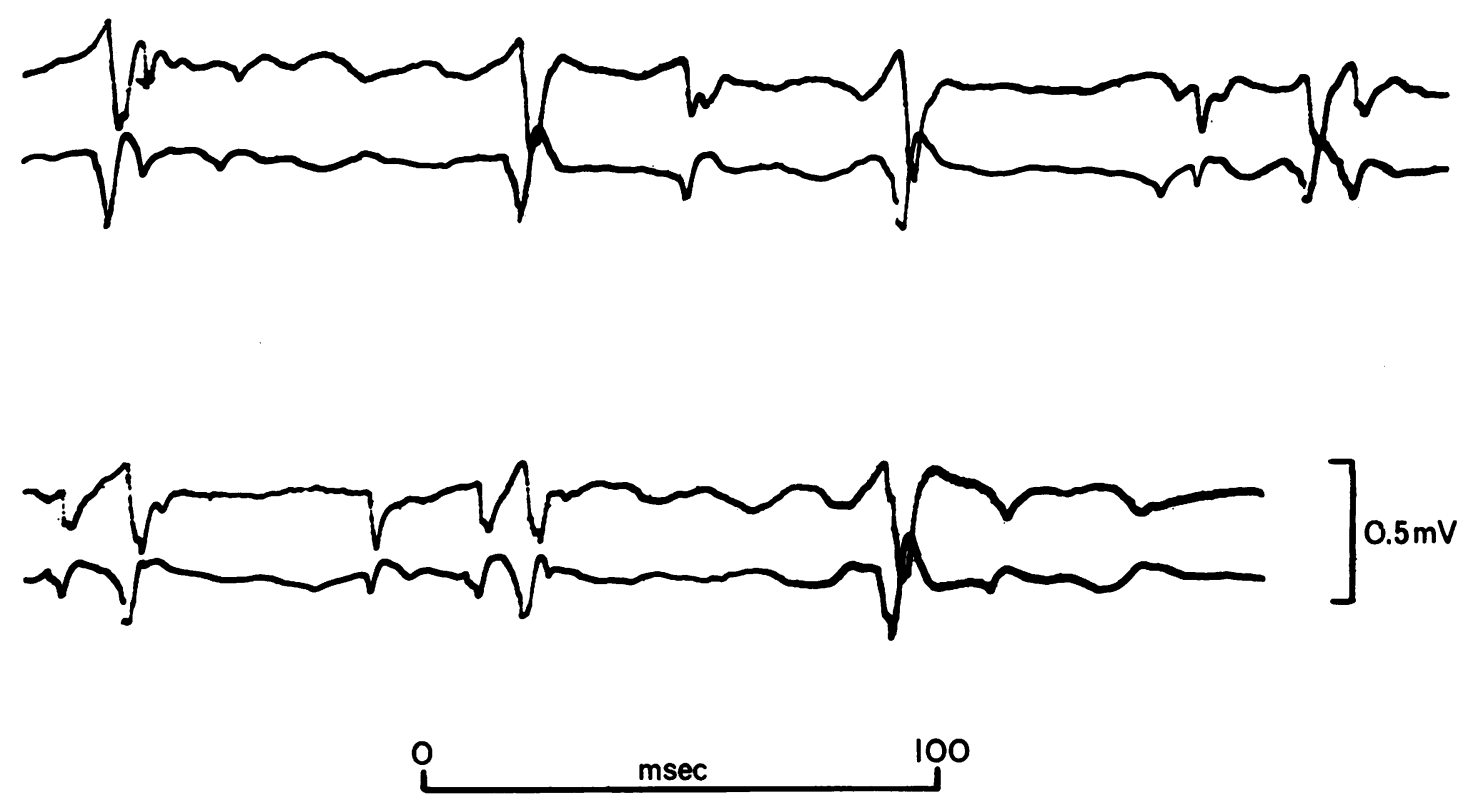

FIG. 3. EMG on slight voluntary contraction of vastus medialis recorded with two concentric needle electrodes arranged along the muscle fibres and separated by $11.5 \mathrm{~cm}$. Muscle fibre conduction velocity had just been measured. The upper and lower traces are continuous.

were at opposite ends of the same muscle fibres. A few milliseconds delay might occur between the two recordings, showing that the end-plate zone was nearer one electrode than the other. However, this delay never approached the latency on direct electrical activation through the stimulating needle, indicating that the endplate zone was some distance from either needle. Assuming linear conduction along the fibres, it was possible in this way to estimate the approximate position of the end-plate zone.

These observations confirm that it is the muscle fibres that are being stimulated by the technique described. Certainly intramuscular nerve fibres can also be stimulated in this way, but this does not usually occur unless the needle is in the region of the end-plate zone. Figure 4 shows the effect of increasing cathodal stimuli delivered to the outer casing of a needle inserted close to the region of the motor point. With the larger stimuli an early EMG response is seen in addition to the usual direct response, and this almost certainly results from stimulating intramuscular nerve bundles.

\section{RESULTS}

VISUAL OBSERVATION DURING MUSCLE PERCUSSION When normal muscle is percussed sharply with a tendon hammer, there is usually an immediate, brief contraction involving muscle fibres passing beneath the site of the blow. This appears to involve the whole length of muscle fibres, for in favourable circumstances an immediate strip of contraction may be seen involving the greater part of the length of the muscle. The duration of the contraction was assessed only visually but did not usually appear to exceed one second in duration, rather shorter than described by Denny-Brown and Pennybacker (1938). The localized contraction with slow spread along the fibres reported by Schiff (1858) and others, and investigated by Denny-Brown and Pennybacker was not observed in the present study on normal young adults

EMG RESPONSE EVOKED BY MUSCLE PERCUSSION It proved impossible to record EMG activity close to the site of percussion because of movement artefact produced by the tap. However, when the needle was inserted $5 \mathrm{~cm}$ or more proximally along the muscle from the percussion site, movement artefact became progressively less apparent. EMG activity could then be recorded regularly provided that the needle was inserted among fibres contracting as a result of the tap. The response consists of a shower of activity (Fig. 5) that commences after a latency corresponding approximately to the known membrane conduction velocity of human muscle fibres over the distance in question (see below). The duration of this 
A

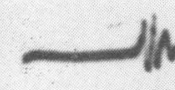

B
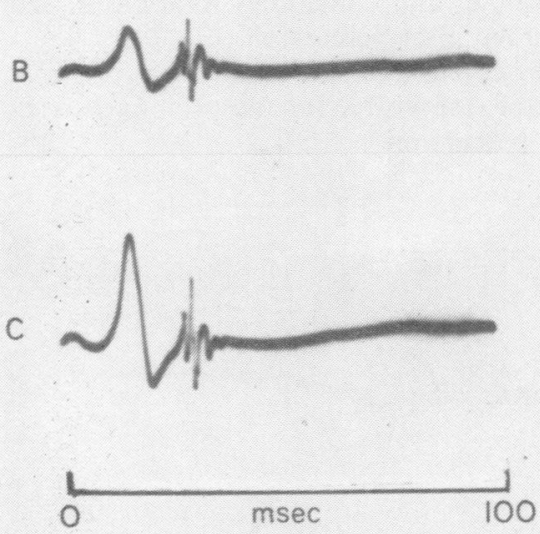

FIG. 4. Evoked EMG responses after stimulation through a concentric needle as in Fig. $1 \mathrm{~B}$ except that the stimulating needle was inserted approximately $3 \mathrm{~cm}$ distal to the motor point, determined beforehand by threshold stimulation with surface electrodes. Increasing cathodal shocks delivered to the outer casing of the needle evoked first a direct response due to stimulation of muscle fibres and then an earlier indirect response to intramuscular nerve stimulation.

response was much longer than expected for a sudden, brief discharge of muscle fibres; depending on the force of percussion it sometimes lasted up to $80 \mathrm{msec}$ or longer. Its amplitude was usually low

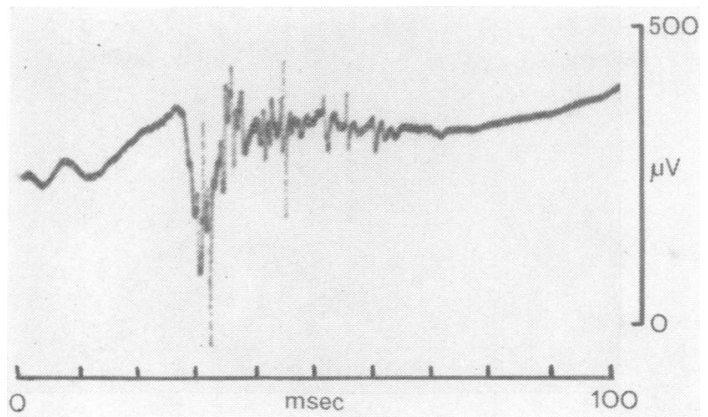

FIG. 5. EMG response recorded with percussion as in Fig. 1A. The concentric recording electrode was inserted $11 \mathrm{~cm}$ proximally along the muscle from the site of percussion. Oscilloscope sweep triggered at moment of impact.
(100 to $300 \mu \mathrm{V}$ ), but some components might reach higher amplitudes when the needle was favourably positioned. The rapid-rise time of these components then suggests that individual muscle fibres or small groups of fibres are being activated rather than motor units. This, coupled with the fact that the response can be elicited with percussion so far distally in the muscle that few if any motor nerve fibres can be present, suggests that the EMG response is due to direct muscle activation. This is confirmed by the observation that the latency of the response always depends upon the distance between percussion site and recording electrode, which corresponds to a muscle membrane conduction velocity of around $4 \mathrm{~m} / \mathrm{sec}$.

On a few occasions, a probable spinal reflex component could be recognized among the prolonged EMG response after percussion (Fig. 6). However, reflex phenomena were not responsible for the prolonged activity and typical responses could be obtained after complete blockade of the nerve to vastus medialis with $2 \%$ xylocaine (Fig. 7), the nerve having been located roughly by surface stimulation and more accurately by stimulating down the needle through which the xylocaine was subsequently injected. Complete nerve blockade was confirmed by the absence of visible contraction and EMG activity on volition or on nerve stimulation above the injection site (see Fig. 1A).

The mechanism of the prolonged activity came to

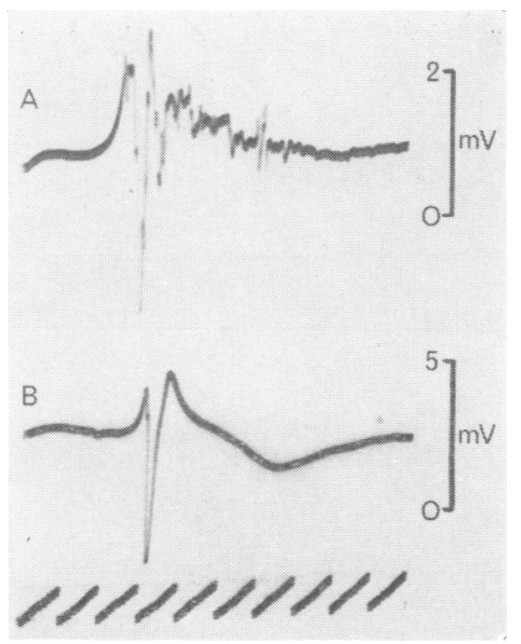

FIG. 6. A. EMG response recorded with percussion as in Fig. 1A. Distance $12 \mathrm{~cm}$. B. EMG response after patellar tendon tap in the same subject. Time scale $10 \mathrm{msec}$ per slope. 


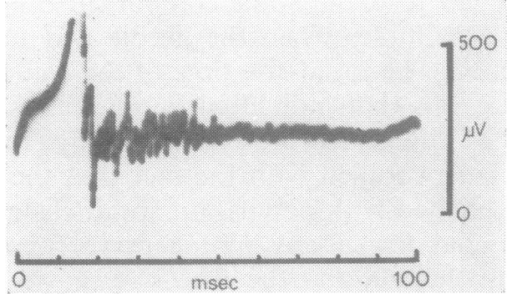

FIG. 7. EMG response recorded with percussion as in Fig. 1A. Distance $12 \mathrm{~cm}$. Complete xylocaine block of nerve to vastus medialis.

light in occasional records obtained during the early percussion experiments. In these records it appeared that repetitive firing was occurring in some of the muscle fibres activated by percussion. Initially this was not easy to demonstrate with an ordinary concentric needle electrode, because the effect of percussion involved many muscle fibres giving rise to an EMG interference pattern in most instances, but once the importance of percussing at the very distal end of the muscle where it tapers to its insertion was recognized it was seen more commonly. Relatively few muscle fibres may be activated in this way and many records have now been obtained where a particular component can be seen discharging repetitively. Two examples are shown in Fig. 8. (Fig. 8B is of poor photographic quality but is included because the experiment was performed after complete blockade of the nerve to vastus

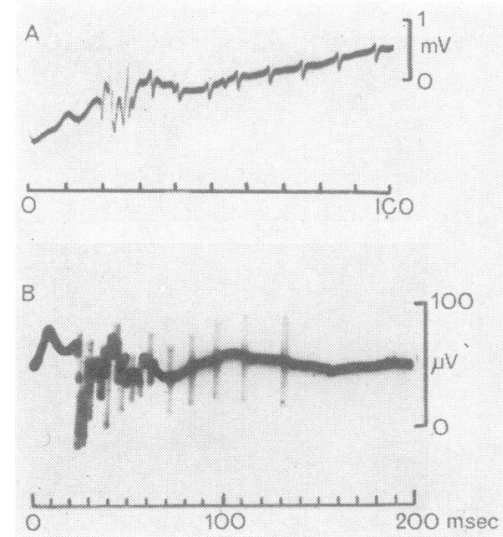

FIG. 8. EMG responses recorded with percussion as in Fig. 1A, showing repetitive potentials. The records shown are taken on different occasions in the same subject. Distances $9 \mathrm{~cm}$ in A, $10 \mathrm{~cm}$ in B. In B, the nerve to vastus medialis was blocked with xylocaine. medialis with $2 \%$ xylocaine). Repetitive firing after $\frac{Z}{\mathcal{D}}$ percussion has been confirmed in experiments performed with an Ekstedt needle, which allows more discrete recording of individual components in what would be an uninterpretable interference pattern with an ordinary concentric electrode. Single, double, and sometimes multiple discharges were recorded after percussion (Fig. 9).

It is suggested that such repetitive firing is the major factor responsible for the prolonged response following percussion.

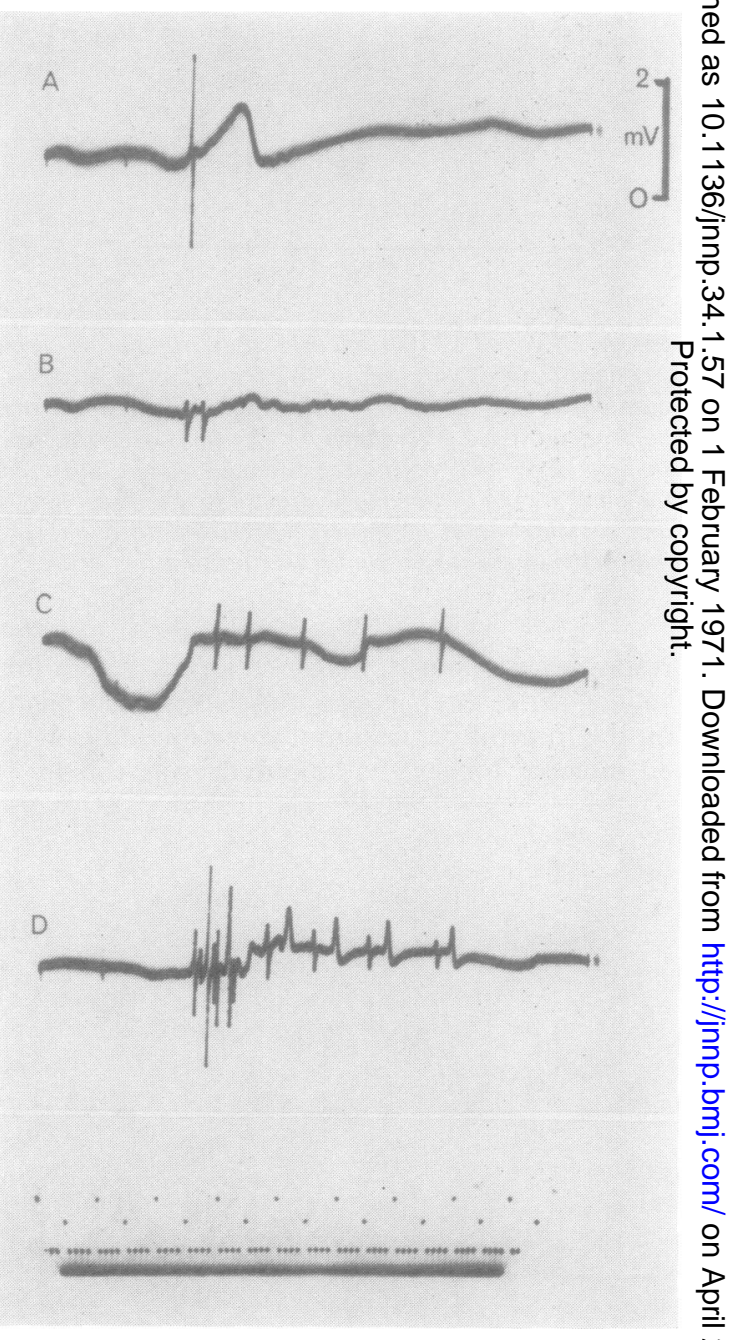

FIG. 9. Single (A), double (B) and multiple discharges $\mathrm{N}$ (C and $\mathrm{D})$ after percussion. Recording as in Fig. 1A but with an Ekstedt needle. Distance $10 \mathrm{~cm}$. Time marker shows 1, 5, and 10 msec marks. 
EMG INSERTIONAL ACTIVITY The immediate question that then arises is whether this repetitive firing merely represents 'insertional' activity arising at the recording needle. (In the present context, insertional activity refers to EMG potentials arising at the point of contact between the recording needle and muscle fibres which is caused by the mechanical effect of movement between the two.) According to Kugelberg and Petersen (1949) insertion or movement of a needle provokes repetitive firing at rates up to $200 /$ sec, the discharge outlasting the actual shifting of the needle by a few tenths of a second and stopping either abruptly or with a few beats at a lower rate.

There are two possible sources of insertional activity to be considered in the present experiments. Firstly, movement of the tissues produced by the percussion inevitably reaches the recording needle and might give rise to insertional activity; certainly it may cause low frequency, movement artefact in the recordings. Secondly, it is possible that movement of the contracting fibres themselves against the tip of the needle is sufficient to provoke insertional activity even if transmission of the percussion wave does not. Further experiments have disproved both these possibilities:

Moving the site of percussion a centimetre or so to one side activates an adjacent strip of muscle and no EMG response is recorded with the needle positioned for the initial site. The transmitted movement artefact is little if at all altered by this manoeuvre, since the distance between percussion and recording sites is not changed significantly. In contrast, percussion 3 to $4 \mathrm{~cm}$ to one side of the recording needle produces gross movement artefact but does not regularly cause EMG activity. Furthermore, when the extreme distal end of vastus medialis is percussed there may be remarkably little movement artefact with the recording needle in its usual site. The fibres that are then activated form the most superficial part of the muscle more proximally and it is only with the needle point in this most superficial part of the muscle that a good EMG response can be recorded. Movement artefact can be further reduced by pressing the ulnar margin of the hand transversely between percussion and recording sites; the EMG response can still be recorded.

The second possibility, that insertional activity arises because of the movement of contracting muscle fibres, seems very unlikely. Such EMG activity does not usually accompany voluntary or electrically evoked contraction. It is true that with a concentric needle electrode in vastus medialis, insertional activity may sometimes occur with nerve stimulation in mid-thigh (Fig. 10) or during the elicitation of a patellar reflex, but the contraction in these circumstances can be large and cause profound shifting of

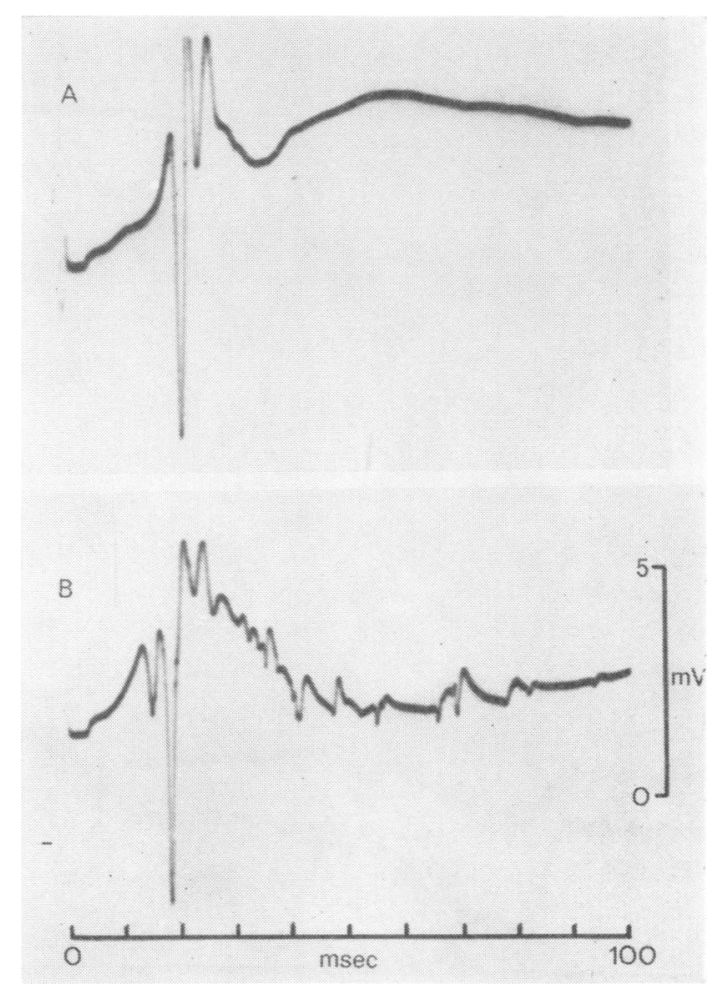

FIG. 10. A. EMG response with a concentric needle electrode in vastus medialis when its nerve is stimulated maximally with surface electrodes in mid-thigh. B. With the needle position slightly adjusted a train of insertional activity followed the main evoked response.

the needle position, unlike the effects of careful distant percussion. When such insertional activity does occur, the initial deflection is negative-a point of considerable importance as will now be shown.

INITIAL POLARITY OF MUSCLE FIBRE DISCHARGES Buchthal and Rosenfalck (1966)have pointed out that the fibrillation potentials of denervated muscle have an initial negative deflection when recorded in the end-plate zone (where they are said to arise) and an initial positive deflection when recorded elsewhere in the muscle. They argue that an initial negative deflection indicates conduction away from the electrode and an initial positive deflection indicates conduction towards the electrode. The same principle can be applied to the present study. Experiments have shown that the individual potentials of insertional activity provoked by needle movement usually have an initial sharp negative component followed by a smaller, longer positive phase (Fig. 11A). The initial negative component indicates that the poten- 


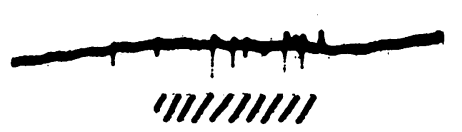

A

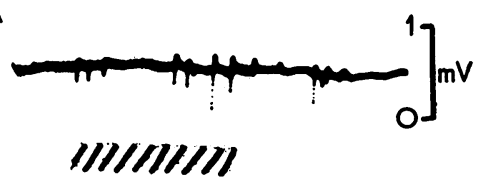

C
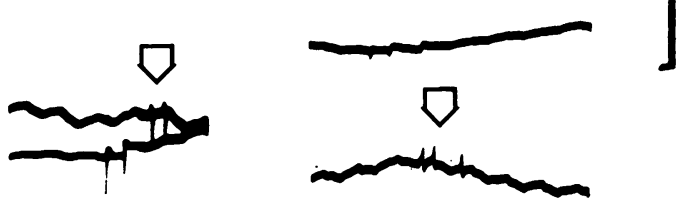

B
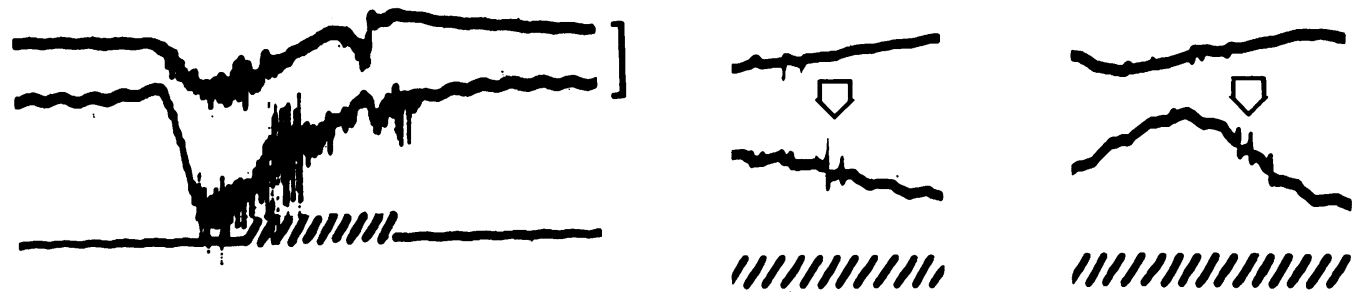

IIIIIIIIIIIIIII

FIG. 11 A. EMG 'insertional activity' provoked by needle movement in vastus madialis. As in other records shown, a upward deflexion is positive and a downward deflexion negative. B. EMG 'insertional activity' provoked by needle move ment (upper record) is recorded $10.5 \mathrm{~cm}$ proximally along the muscle with a second concentric needle electrode (lower record). Cal $1 \mathrm{mV}$ for upper record, $200 \mu \mathrm{V}$ for lower record. C. Four examples of propagated EMG insertional activity to show initial positive polarity when recorded at a distant point along the muscle fibres. This was difficult to demonstrate and the records, though clearly showing the point in question are disturbed by mains and movement artefact. Arrows point to the recorded potentials. The other record is from the needle at which the activity is arising. Cal $1 m V$ for electrode of origin, $200 \mu V$ for 'recording' electrode. Time scale $10 \mathrm{msec}$ per slope for all records.

tials arise at the needle and are conducted away from it. Insertional activity is propagated along the muscle membrane at a normal rate and can be recorded with a second needle electrode carefully positioned (Fig. 11b). It proved difficult to record individual potentials from both needles at once but when this was achieved the initial deflection was negative in the needle in which the activity was arising and positive in the distant needle (Fig. 11c). When the EMG activity evoked by distant percussion is examined, it is found that the individual potentials are usually sharply diphasic and have an initial positive polarity-for example, Figs. 5, 8, and 9). This initial positive polarity provides strong evidence that the EMG activity evoked by per- cussion arises at the site of the tap and is propagated towards the recording needle. Therefore it cannot represent insertional activity arising at the electrode tip.

MUSCLE FIBRE STIMULATION AND MUSCLE MEMBRANE CONDUCTION VELOCITY The EMG responses recorded when muscle fibres were stimulated through a needle were always of much shorter duration than those evoked by percussion, even when many fibres were activated by delivering large shocks through the casing of the needle-for example, Fig. 4. However the latencies of the two types of response over similar distances always corresponded.

Muscle membrane conduction velocity was 
measured in a total of 22 experiments on the two vasti medialis of four subjects. Mean propagation velocity at threshold stimulation-that is, for the first 'spike' appearing - was $3.95 \mathrm{~m} / \mathrm{sec}$, SD 0.41 . The average distance studied was $9.6 \mathrm{~cm}$ and the mean amplitude of the first spike at threshold was $137 \cdot 3 \mu \mathrm{V}$, SD $91 \cdot 3$.

This figure for propagation velocity is remarkably close to that of $4.02 \mathrm{~m} / \mathrm{sec}$ calculated for biceps brachii by Buchthal, Guld, and Rosenfalck (1955). The values obtained by Stålberg (1967) with a multielectrode in different muscles during voluntary contraction were rather lower $(3.69 \mathrm{~m} / \mathrm{sec}$ in biceps brachii, $3.15 \mathrm{~m} / \mathrm{sec}$ in extensor digitorum communis, and $3.39 \mathrm{~m} / \mathrm{sec}$ in quadriceps femoris) but this appears to relate to the technique used, which is influenced by the fall in velocity that Stålberg demonstrates to occur during sustained voluntary contraction.

POST-PERCUSSIVE FASCICULATION In two of the subjects studied, heavy percussion occasionally produced a second, additional type of response, consisting of random fasciculation which may appear immediately after the primary twitch or may be delayed a second or more. It has the appearance of true fasciculation and is clearly of neurogenic origin. Its extent varies from an isolated twitch of a single fasciculus to, occasionally, more extensive fasciculation affecting a large area of muscle and lasting several seconds. Sometimes the same fasciculus may be seen twitching in a recurrent, though irregular manner. The involved fasciculi are not necessarily those immediately under the site of percussion.

Post-percussive fasciculation was more easily evoked and usually more extensive on percussing over the motor point than over other parts of the muscle and it was rarely seen when percussing the muscle distally. This suggests, as would be expected, that it is caused by mechanical stimulation of intramuscular nerve fibres. In contrast, the primary twitch evoked by percussion (direct muscle response) was not more easily elicited by percussion over the motor point.

In the experiments in which the nerve to vastus medialis was blocked with local anaesthetic there was no detectable difference in the ease with which post-percussive fasciculation could be elicited in vastus medialis compared with before the block. In two further experiments the median nerve was blocked at the wrist by injecting $2 \mathrm{ml} .2 \%$ xylocaine into the carpal tunnel. Fasciculation could still be induced in abductor pollicis brevis (where it may be particularly prominent, perhaps because of the high content of motor nerve fibres to the small hand muscles), although in one experiment it may have been less pronounced than before the block.

\section{DISCUSSION}

The present study shows that muscle percussion in the normal, young adult initiates a brief burst of action potentials in muscle fibres passing beneath the site of the tap. By directly stimulating the muscle fibres it causes a repetitive discharge, usually lasting about 30 to $80 \mathrm{msec}$, which is propagated along the fibres and is seen as an immediate, brief contraction of that part of the muscle. With percussion close to the motor point, delayed fasciculation may also result but this is a different phenomenon which depends upon intramuscular motor nerves, and it is discussed in the appropriate section. Other phenomena that have been described with percussion, such as a local contraction confined to the percussion site and the slowly propagated, electrically silent waves of contraction studied in cachexia by DennyBrown and Pennybacker (1938), were not observed in the present subjects. It might be questioned whether the wasting of cachexia unmasks slowly transmitted waves that in healthy subjects are concealed by subcutaneous tissue. This seems most unlikely, since careful scrutiny of the shaft of an EMG needle recording at a distance along muscle fibres activated by percussion failed to reveal any late movement. Yet fine movement of the recording needle proved a most sensitive visual index of contraction when the muscle fibres were stimulated electrically through a distant needle.

Initially it seemed possible that the repetitive discharge seen with percussion was merely insertional EMG activity arising at the recording needle because of movement of the tissues transmitted from the percussion site. Reasons have been given why this is not so, but nevertheless insertional activity and the response to percussion show marked similarities and they may simply be different manifestations of the same basic sensitivity of muscle to mechanical stimuli, the former being caused by the stimulus of needle movement, the latter by the more massive stimulus of percussion. Both are characterized by repetitive firing at rates often well in excess of $100 / \mathrm{sec}$, which tend to slow before stopping completely. The slowing is more obvious with percussion (Fig. 8) than with insertional activity but in the latter case the issue is complicated by the fact that the recording electrode is necessarily moving. Not only does this mean that what is recorded changes during the movement but it also means that interpretable records-that is, records in which the same component can be seen firing repetitively-are likely to be obtained only with slower and gentler movements 
of the needle; with large or abrupt movements artefact often renders them uninterpretable. Recording is thus more difficult and the stimulus not so instantaneous with records of insertional activity. Irregularities in discharge rate are common but these may reflect irregularities in the stimulus (erratic movement between the needle tip and the muscle) rather than any property of the muscle fibre itself. Nevertheless, when individual potentials can be recognized, progressive slowing of their discharge rate may be evident (Fig. 12).

The different initial polarity of these two types of potential is to be expected from their different sites of origin but their configurations are also different and this too must be explained. As already stated the potentials of insertional activity most commonly consist of a sharp negative spike followed by a longer, low amplitude positive phase, while with percussion the individual components appear to be mostly of sharply diphasic waveform, usually resembling muscle fibre potentials. An important factor in accounting for this difference may be that in insertional activity the depolarization is arising $a t$ the electrode tip and therefore conducting away from this point while potentials propagated from the percussion site are approaching, reaching, and then passing away from the recording needle. Moreover the needle tip is in actual contact with the depolarizing muscle fibres and therefore in a peculiar position, perhaps not regularly shared in the case of propagated potentials approaching from a distance. The marked difference in shape of the two types of waveform should not therefore unfavourably influence attempts to unify these two forms of activity.

It is interesting to extend this comparison to include the myotonias, where the mechanical sensitivity of muscle is markedly increased and prolonged, both in the responses to percussion and to needle movement. The initial effect of the mechanical stimulus is probably the same as in the normal subject except that the mechanical threshold may be lower. The result is propagated depolarization in a number of muscle fibres. However, while in the normal subject percussion causes repetitive firing in only a proportion of these fibres and for only a fraction of a second, in the myotonias the response involves many more fibres and lasts many times longer. The same applies for myotonic discharges caused by needle movement; these may reach a frequency of $200 / \mathrm{sec}$ - for example, Floyd et al., 1955-similar to that reached in normal insertional activity. However, the myotonic discharge sometimes continues at a declining frequency for several seconds, again contrasting with the brief insertional response in the normal subject. The responses to mechanical stimulation in myotonia can thus be regarded as an extension of what occurs in the normal subject, in the same way that DennyBrown and Nevin (1941) regarded the repetitive EMG response after a single nerve stimulus in myotonia as an extension of the brief, repetitive discharge that can occasionally be seen in normal muscle (Eccles and O'Connor, 1938).

My thanks are due to Dr. R. G. Willison for allowing meñ to use his Ekstedt needle and apparatus at the Nationa Hospital, Queen Square. I am also grateful for his com- $-2-$ ments, and those of Dr. P. M. Fullerton and Dr. C. D. $\mathbb{Q}$ Marsden in the construction of this paper.

REFERENCES

Brown, G. L., and Harvey, A. M. (1939). Congenital myotonia in the goat. Brain, 62, 341-363.

Buchthal, F., Guld, C., and Rosenfalck, P. (1955). Propagation velocity in electrically activated muscle fibres in man. Acta physiol. scand., 34, 75-89.

Buchthal, F., and Rosenfalck, P. (1966). Spontaneous electrical activity of human muscle. Electroenceph. clin. Neurophysiol., 20, 321-336.

Coërs, C., and Woolf, A. L. (1959). The Innervation of Muscle: a Biopsy Study. Blackwell: Oxford.
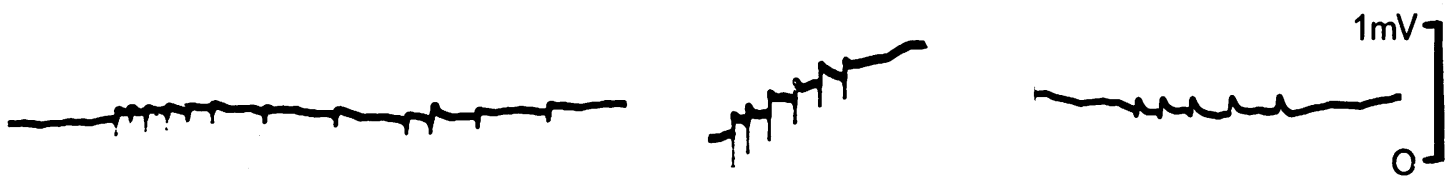

\section{IIIIIIIIIIIIIIIIIIIIIIIIII}
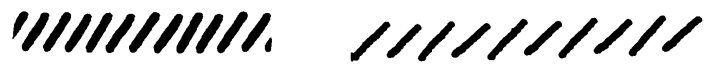

FIG. 12. Slowing discharge rate in insertional EMG activity. In the first example there is a single, different potential superimposed upon the train of discharges. Time scale 10 msec per slope. 
Curschmann, H. (1905). Untersuchungen über die idiomuskuläre Übererregbarkeit (idiomuskuläre kontraktion Schiffs). Dtsch. Z. Nervenheilk., 28, 361-403.

Denny-Brown, D., and Pennybacker J. B. (1938). Fibrillation and fasciculation in voluntary muscle. Brain, 61, 311-334.

Denny-Brown, D., and Nevin, S. (1941). The phenomenon of myotonia. Brain, 64, 1-18.

Eccles, J. C., and O'Connor, W. J. (1938). Responses evoked by a nerve volley in mammalian striated muscle. J. Physiol. (Lond.), 94, 7-9P.

Ekstedt, J. (1964). Human single muscle fibre action potentials. Acta physiol. scand., 61, Suppl. 226.

Floyd, W. F., Kent, P., and Page, F. (1955). An electromyographic study of myotonia. Electroenceph. clin. Neurophysiol., 7, 621-630.
Kugelberg, E., and Petersen, I. (1949). 'Insertion activity' in electromyography with notes on denervated muscle response to constant current. J. Neurol. Neurosurg. Psychiat., 12, 268-273.

Salick A. I., and Pearson, C. M. (1967). Electrical silence of myoedema. Neurology (Minneap.), 17, 899-901.

Schiff, J. M. (1858). Muskel und Nerven-physiologie, Schauenberg's Cyclus, Th. 9, Bd. 1, Lahr. Quoted by Denny-Brown and Pennybacker (1938).

Stålberg, E. (1967). Propagation velocity in human muscle fibres in situ. Acta physiol. scand., 70, Suppl. 287.

Walthard, K. M., and Tchicaloff, M. (1961). Motor points. In Electrodiagnosis and Electromyography, pp. 153-170, 2nd edn. Edited by S. Licht. E. Licht: New Haven. 